\title{
LEFT REGULAR BANDS OF GROUPS OF LEFT QUOTIENTS
}

\author{
by ABDULSALAM EL-QALLALI
}

(Received 30 May, 1989)

Introduction. In this paper we characterize semigroups $S$ which have a semigroup $Q$ of left quotients, where $Q$ is an $\mathscr{R}$-unipotent semigroup which is a band of groups. Recall that an $\mathscr{R}$-unipotent (or left inverse) semigroup $S$ is one in which every $\mathscr{R}$-class contains a unique idempotent. It is well-known that any $\mathscr{R}$-unipotent semigroup $S$ is a regular semigroup in which the set of idempotents is a left regular band in that $e f e=e f$ for any idempotents $e, f$ in $S$. $\mathscr{R}$-unipotent semigroups were studied by several authors, see for example [1] and [13]. Bailes [1] characterized $\mathscr{R}$-unipotent semigroups which are bands of groups. This characterization extended the structure of inverse semigroups which are semilattices of groups. Recently, Gould studied in [7] the semigroup $S$ which has a semigroup $Q$ of left quotients where $Q$ is an inverse semigroup which is a semilattice of groups.

Many definitions of semigroups of quotients have been proposed and studied. For a survey, the reader may consult Weinert's paper [14]. These definitions have been motivated by corresponding definitions in ring theory. In this paper we are concerned with a concept of semigroups of left quotients adopted by Fountain and Petrich [5]. The definition proposed there is restricted to completely 0 -simple semigroups of left quotients. The idea is that a completely 0 -simple semigroup $Q$ containing a subsemigroup $S$ is a semigroup of left quotients of $S$ if every element $q$ in $Q$ can be written as $q=a^{-1} b$ for some elements $a, b$ in $S$ with $a^{2} \neq 0$ and $a^{-1}$ the inverse of $a$ in the group $\mathscr{H}$-class $H_{a}$ of $Q$. In this case $S$ is also called $a$ left order in $Q$. This definition and its dual were used in [5] to characterize semigroups $S$ which have a completely 0 -simple semigroup of quotients. An extension of this definition was used in [6] to obtain necessary and sufficient conditions for a semigroup $S$ to have a bisimple inverse $\omega$-semigroup of left quotients. This extended definition was also used in [7] to characterize semigroups $S$ which have a semigroup $Q$ of left quotients where $Q$ is an inverse semigroup which is a semilattice of groups. In this paper we consider the corresponding problem for $\mathscr{R}$-unipotent semigroups which are bands of groups.

After preliminary results, we obtain in Section 2 , the necessary and sufficient conditions for a semigroup $S$ to have a semigroup $Q$ of left quotients where $Q$ is an $\mathscr{R}$-unipotent semigroup which is a band of groups. This result will be used in Section 3 together with the characterization of $\mathscr{R}$-unipotent semigroups which are bands of groups in terms of spined products to obtain an alternative structure for a semigroup $S$ to have a left regular band of groups as a semigroup of left quotients. Section 4 is devoted to the case where the left orders are in a class of $\mathscr{R}^{*}$-unipotent semigroups.

We use the notation and terminology of Howie [9]. Other undefined terms can be found in Fountain's paper [4].

1. Preliminaries. Recall that for a semigroup $S$, any two elements $a, b$ in $S$ are $\mathscr{R}^{*}$-related if they are related by Green's relation $\mathscr{R}$ in some oversemigroup of $S$. The following Lemma from [10] and [11] gives an alternative definition of $\mathscr{R}^{*}$.

Glasgow Math. J. 33 (1991) 29-40. 
LeMma 1.1. For any two elements $a, b$ in a semigroup $S$, the following statements are equivalent:

(i) $a \mathscr{R}^{*} b$ in $S$

(ii) $s a=t a \Leftrightarrow s b=t b ; \forall s, t \in S^{1}$.

The following Corollary is a consequence of Lemma 1.1:

COROLlaRY 1.2. If $a$ is an element of a semigroup $S$ and $e$ is an idempotent in $S$, then $a \mathscr{R}^{*} e$ in $S$ if and only if

(i) $e a=a$, and

(ii) $s a=t a \Rightarrow s e=t e ; \forall s, t \in S^{1}$.

The dual relation of $\mathscr{R}^{*}$ is $\mathscr{L}^{*}$. It is easy to see that $\mathscr{R}^{*}$ is a left congruence and $\mathscr{L}^{*}$ is a right congruence. Thus the intersection of $\mathscr{R}^{*}$ and $\mathscr{L}^{*}$ is an equivalence relation denoted by $\mathscr{K}^{*}$. It is evident for $\mathscr{K}=\mathscr{R}, \mathscr{L}$, or $\mathscr{H}$ that $\mathscr{K} \subseteq \mathscr{K}^{*}$ and for any regular elements $a, b$ in a semigroup $S ; a \mathscr{K} b$ follows from $a \mathscr{K}^{*} b$. Therefore $\mathscr{K}=\mathscr{K}^{*}$ on regular semigroups.

As a consequence of Corollary 1.2 and its dual or from [4] we have the following Lemma:

Lemma 1.3. If $e$ is an idempotent of a semigroup $S$, then, $H_{e}^{*}$ is a cancellative monoid.

Moreover, we have as a consequence of Proposition 1.13 of [4]:

LEMMA 1.4. If $e, f$ are $\mathscr{L}$-related idempotents in a semigroup $S$, then $H_{e}^{*} \approx H_{f}^{*}$.

It is known that if $Q$ is an $\mathscr{R}$-unipotent semigroup which is a band of groups, then $Q$ can be written as a disjoint union of groups $G_{\alpha}, \alpha \in Y$, that is, $Q=\bigcup_{\alpha \in Y} G_{\alpha}$, where $Y$ is a band isomorphic to the band of idempotents of $Q$. In particular $Y$ is a left regular; so we may call $Q$ a left regular band of groups. We refer the reader to [1] for further details. As a consequence we have the following Corollary:

COROLlaRY 1.5. Let $Q$ be a left regular band of groups and $E$ be its band of idempotents. Then $Q$ is the left regular band $E$ of the $\mathscr{H}$-classes $H_{e} ; e \in E$ of $Q$.

From [1] we have the following result:

THEOREM 1.6. Let $Q$ be an $\mathscr{R}$-unipotent semigroup. Then:

(a) $Q$ is a union of groups if and only if $\mathscr{R}=\mathscr{H}$ in $Q$.

(b) $Q$ is a band of groups if and only if $\mathscr{R}$ is a congruence on $Q$.

The central concept in our work is the concept of semigroups of left quotients. We say an oversemigroup $Q$ of a semigroup $S$ is a semigroup of left quotients of $S$ if for any element $a$ in $S, a$ is in a subgroup of $Q$ whenever $a \mathscr{H}^{*} a^{2}$ in $S$, and for any element $q$ of $Q$, there exist $a, b$ in $S$ such that $q=a^{-1} b$ where $a^{-1}$ is the inverse of $a$ in a subgroup of $Q$. Similar definitions were adopted in [5], [6], [7] and [8]. If $Q$ is a semigroup of left quotients of a semigroup then $S$ is said to be a left order in $Q$. Our investigation will be on the light of left regular bands of right reversible, cancellative semigroups. We recall from [2] that a semigroup $S$ is right reversible if $S a \cap S b \neq \varnothing$ for all $a, b$ in. $S$, that is, for any $a, b$ in $S$, there exist $x, y$ in $S$ with $x a=y b$.

For cancellativity, we conclude from [7] the following Lemma: 
Lемма 1.7. If $S$ is a semilattice of cancellative semigroups, then $\mathscr{H}^{*}$ is the greatest band congruence on $S$ all of whose classes are cancellative.

2. Left orders in a band of groups. Let $Q$ be an $\mathscr{R}$-unipotent semigroup with set of idempotents $E$. Recall that $E$ is a left regular band and every $\mathscr{R}$-class in $Q$ contains a unique idempotent. Consider $Q$ to be a band $Y$ of groups $G_{\alpha} ; \alpha \in Y$, where for any $\alpha, \beta \in Y, G_{\alpha} \cap G_{\beta}=\varnothing$ if $\alpha \neq \beta$ and $Q=\bigcup_{\alpha \in Y} G_{\alpha} ; G_{\alpha} G_{\beta} \subseteq G_{\alpha \beta}, E \approx Y$, and $Y$ is a left regular band. Moreover, by Corollary 1.5, the groups $G_{\alpha}, \alpha \in Y$ are taken to be the $\mathscr{H}$-classes of $Q$. Now let $S$ be a semigroup which is a left order in $Q$. Put $S_{\alpha}=S \cap G_{\alpha}$ for any $\alpha \in Y$. From Theorem 1.6, $\mathscr{H}$ is a congruence on $Q$, so that from Propositions (2) and (4) of [8], $S_{\alpha} \neq \varnothing$ for all $\alpha \in Y$. Clearly, for any $\alpha$ in $Y, S_{\alpha}$ is a subsemigroup of $S$. Since $S_{\alpha}$ is a subsemigroup of the group $G_{\alpha}, S_{\alpha}$ is cancellative. Moreover we have:

LeMma 2.1. For any $\alpha \in Y$, the semigroup $S_{\alpha}$ is a left order in $G_{\alpha}$.

Proof. This is immediate from Propositions (2) and (4) of [8].

Proposition 2.2. The semigroup $S$ is a left regular band $Y$ of right reversible, cancellative semigroups $S_{\alpha}, \alpha \in Y$.

Proof. This follows from Lemma 2.1 and Theorem 1.24 of [2].

The following Corollary is immediate.

Corollary 2.3. If $q \in Q$, then there exist $a, b$ in $S$ with $a \mathscr{H} b$ in $Q$ and $q=a^{-1} b$.

The objective now towards a converse of Proposition 2.2. Let $S$ be a semigroup which is a left regular band $Y$ of right reversible, cancellative semigroups $S_{\alpha}, \alpha \in Y$. By Theorem 1.24 of [2], for each $\alpha \in Y, S_{\alpha}$ has a group $G_{\alpha}$ of left quotients. We may assume $G_{\alpha} \cap G_{\beta}=\varnothing$ for any $\alpha, \beta \in Y, \alpha \neq \beta$. Put $Q=\bigcup_{\alpha \in Y} G_{\alpha}$. Notice that if $b \in S_{\alpha}$ and $c \in S_{\beta}$, then $b c \in S_{\alpha \beta}$ and since $Y$ is a left regular band, $b c b \in S_{\alpha} S_{\beta} S_{\alpha} \subseteq S=S_{\alpha \beta \alpha}=S_{\alpha \beta}$. By right reversibility of $S_{\alpha \beta}$ there exist $x^{\prime}, y^{\prime} \in S_{\alpha \beta}$ with $x^{\prime} b c b=y^{\prime} b c$. Putting $x=x^{\prime} b c, y=y^{\prime} b$. Clearly, $x b=y c, x=x^{\prime} b c \in S_{\alpha \beta} S_{\alpha} S_{\beta} \subseteq S_{\alpha \beta}$ and $y \in S_{\alpha \beta}$. Furthermore, for any $a \in S_{\alpha}, d \in$ $S_{\beta}, x a \in S_{\alpha \beta \alpha}=S_{\alpha \beta}, y d \in S_{\alpha \beta \beta}=S_{\alpha \beta}$ and so $(x a)^{-1} y d$ exists in $G_{\alpha \beta}$.

Now define a product . on $Q$ by

$$
a^{-1} b \cdot c^{-1} d=(x a)^{-1} y d
$$

where, if $a, b \in S_{\alpha} ; c, d \in S_{\beta}$, then $x, y \in S_{\alpha \beta}$ are chosen so that $x b=y c$.

Since $Y$ is a left regular band,

$$
x a \in S_{\alpha \beta} S_{\alpha} \subseteq S_{\alpha \beta \alpha}=S_{\alpha \beta} \text { and }(x a)^{-1} \in G_{\alpha \beta}
$$

also

$$
y d \in S_{\alpha \beta} S_{\beta} \subseteq S_{\alpha \beta \beta}=S_{\alpha \beta} \text { and } y d \in G_{\alpha \beta} .
$$

Therefore, the product $(x a)^{-1} y d$ is taken as the product in $G_{\alpha \beta}$. We notice that the property of left regularity of the band $Y$ together with right reversibility and cancellatively of $S_{\alpha}, \alpha \in Y$ are sufficient to carry out the proof of [7] for $Q$ to be a groupoid under the given product, so we omit it here. To prove that product is associative we need the following Lemma. 
Lemma 2.4. If $\alpha \in Y$ and $a, b$ are elements of $S_{\alpha}$, then $a \mathscr{R}^{*} b$ in $S$.

Proof. Let $\alpha \in Y, a, b \in S_{\alpha}$ and $s \in S_{\lambda}, t \in S_{\mu}$ form some $\lambda, \mu$ in $Y$ with $s a=t a$. Then $S_{\lambda \alpha}=S_{\mu \alpha}$. Put $\beta=\lambda \alpha=\mu \alpha$. Since $s a, t a$ in $S_{\beta}$, then by right reversibility of $S_{\beta}$, there exist $m, n$ in $S_{\beta}$ such that $m s a=n t a$. Notice that

$$
m s \in S_{\lambda \alpha} S_{\lambda} \subseteq S_{\lambda \alpha \lambda}=S_{\lambda \alpha}=S_{\beta} ; \quad n t \in S_{\mu \alpha} S_{\mu} \subseteq S_{\mu \alpha \mu}=S_{\mu \alpha}=S_{\beta}
$$

and again by right reversibility of $S_{\beta}$, there exist $u, v$ in $S_{\beta}$ with $u m s=v n t$ and umsa = vnta. Since um, sa, $v n$ and $t a$ are in $S_{\beta}, s a=t a$ and $S_{\beta}$ is cancellative, then $u m=v n$ ( $=k$, say). It follows that $k s=k t$ and $k s b=k t b$. Since $k, s b$ and $t b$ are in $S_{\beta}$. Then, by cancellativity in $S_{\beta}$ we obtain $s b=t b$.

Now consider the case where $t$ is 1 and assume $s a=1$. $a$ for some $s \in S_{\lambda}$. It follows that $S_{\lambda \alpha}=S_{\alpha}$ and there exist $m, n \in S_{\alpha}$ such that msa=na. Notice that $\alpha=\lambda \alpha$, $\alpha=\alpha \lambda \alpha=\alpha \lambda$ and $m s \in S_{\alpha \lambda}=S_{\alpha}$. By reversibility of $S_{\alpha}$, there exist $u, v \in S_{\alpha}$ with $u m s=v n$. Thus umsa = vna. But $s a=a$ in $S_{\alpha}$ and $u m$, vn $\in S_{\alpha}$, then by cancellativity in $S_{\alpha}$, we get $u m=v n$ (=k, say). It follows that $k s=k$ and $k s b=k b$. Since $k, s b$ and $b$ are in $S_{\alpha}, s b=b=1 . b$.

The case where $s$ is 1 is similar.

We conclude that for any $s, t \in S^{\mathbf{1}}, s a=t a$ implies $s b=t b$.

Similarly, $s b=t b$ implies $s a=t a$. Therefore, by Lemma $1.1, a \mathscr{R}^{*} b$ in $S$.

Corollary 2.5. $a \mathscr{H}^{*} a^{2}$ for any element $a$ in $S$.

Proof. Let $a \in S_{\alpha}, s \in S_{\lambda}, t \in S_{\mu}$ with $a^{2} s=a^{2} t$. It is clear that $a^{2} \in S_{\alpha}$ and $\alpha \lambda=\alpha \mu$ ( $=\gamma$, say). Choose $k \in S_{\gamma}$ and write $k a$. as $=k a$. at. Notice that

$$
k a \in S_{\alpha \lambda} S_{\alpha} \in S_{\alpha \lambda \alpha}=S_{\alpha \lambda}=S_{\gamma} ; \text { as, at } \in S_{\gamma}
$$

and $S$ is cancellative. Hence $a s=a t$.

Now consider the case where $t$ is 1 and assume $a^{2} s=a^{2} .1$ for some $s \in S_{\lambda}$. It follows that $S_{\alpha \lambda}=S_{\alpha}$ and $a$. as $=a$. $a$ in $S_{\alpha}$, $a$, as $\in S_{\alpha}$. By cancellativity in $S_{\alpha}$ we get $a s=a .1$.

The case where $s$ is 1 is similar.

Hence for any $s, t \in S^{1} ; a^{2} s=a^{2} t$ implies $a s=a t$.

It is obvious that $a s=a t$ implies $a^{2} s=a^{2} t$. Therefore by the dual of Lemma $1.1 ; a \mathscr{L}^{*} a^{2}$ in $S$. But $a \mathscr{R}^{*} a^{2}$ in $S$ by Lemma 2.4. Hence $a \mathscr{H}^{*} a^{2}$ in $S$.

Returning now to the product of $Q$, the associativity of the product of $Q$ in the inverse semigroup case was proved in [7] by using only the property that $a \mathscr{R}^{*} b$ in $S$ for any $a, b \in S_{\alpha} ; \alpha \in Y$. Therefore, we can see by Lemma 2.4 that the product of $Q$ is associative by a similar proof to that in [7]. Moreover, it can be seen that the product of $Q$ is an extension of that in $S$. It is immediate from the definition of the product of $Q$, that $G_{\alpha} G_{\beta} \subseteq G_{\alpha \beta}$ for any $\alpha, \beta \in Y$. Therefore $Q$ is a left regular band of groups $G_{\alpha}$; $\alpha \in Y$. From its construction, $Q$ is a semigroup of left quotients of $S$. In conclusion we have established the following result.

THEOREM 2.6. A semigroup $S$ has a left regular band of groups as a semigroup of left quotients if and only if $S$ is a left regular band of right reversible, cancellative semigroups.

Theorem 2.6 shows that, if $S$ is a left regular band of right reversible, cancellative semigroups, then for any decomposition of $S$ as a left regular band $Y$ of right reversible, 
cancellative semigroups, we can construct a semigroup $Q$ of left quotients of $S$ where $Q$ is a left regular band $Y$ of groups. Neither the decomposition of $S$ nor the construction of $Q$ is unique (see [7]). In order to overcome this problem of uniqueness in the inverse semigroup case, the notion of stratified semigroup of left quotients was introduced in [7] as follows:

Let $Q$ be an oversemigroup of semigroup $S$. Then $Q$ is a stratified semigroup of left quotients of $S$ if:

(i) for any elements $a, b$ of $S$, $a \mathscr{R} b$ in $Q$ if and only if $a \mathscr{R}^{*} b$ in $S$ $a \mathscr{L} b$ in $Q$ if and only if $a \mathscr{L}^{*} b$ in $S$

(ii) every element $a$ of $S$ is in a subgroup of $Q$ whenever a $\mathscr{L}^{*} a^{2}$ in $S$

(iii) every element of $Q$ can be written as $a^{-1} b$ where $a, b \in S$ and $a \mathscr{R} b$ in $Q$.

If $Q$ is a left regular band $Y$ of groups that is also a stratified semigroup of left quotients of a semigroup $S$, then $Q$ is, clearly, a left regular band of groups of left quotients of $S$. In this case $Q$ is unique up to isomorphism (Theorem 4.1 of [7]).

Proposition 2.7. Let $Q$ be a left regular band of groups of left quotients of a semigroup $S$ such that for any elements $a, b$ in $S, a \Re^{*} b$ in $S$ implies $a \mathscr{R} b$ in $Q$ and that $a \mathscr{L}^{*} b$ in $S$ implies $a \mathscr{L} b$ in $Q$. Then $Q$ is a stratified semigroup of left quotients of $S$.

Proof. Let $Q$ and $S$ be as given. As $Q$ is an oversemigroup of $S$, it follows that for any $a, b$ in $S, a \mathscr{R} b$ in $Q$ implies $a \mathscr{R}^{*} b$ in $S$ and $a \mathscr{L} b$ in $Q$ implies $a \mathscr{L}^{*} b$ in $S$. Thus condition (i) for a stratified semigroup of left quotients of $S$ holds. Condition (i) and Corollary 2.5 imply $a \mathscr{H} a^{2}$ in $Q$ so that $a$ is in a subgroup of $Q$ for any $a$ in $S$, and condition (ii) holds. Condition (iii) holds by Corollary 2.3 . Hence the result.

3. Punched spined products. In this section we provide an alternative characterization of a semigroup $S$ which has a semigroup $Q$ of left quotients where $Q$ is a left regular band of groups. This characterization will be in terms of spined products. Recall that, if $E$ is a band and $M$ is a semigroup with a semilattice congruence $\tau$ and a semigroup isomorphism $\Phi: E / \varepsilon \rightarrow M / \tau$ where $\varepsilon$ is the minimum semilattice congruence on $E$, then the subdirect product

$$
P=\left\{(e, x) \in E \times M: e \varepsilon^{\natural} \Phi=x \tau^{\natural}\right\}
$$

is called a spined product of $E$ and $M$. A subsemigroup of $P$ that is also a subdirect product of $E$ and $M$ is called a punched spined product of $E$ and $M$. The aim of this section is to prove that the left orders characterized in Section 2 are in fact punched spined products.

Let $Q$ be an $\mathscr{R}$-unipotent semigroup and $E$ be its band of idempotents. Let $\varepsilon$ be the minimum semilattice congruence on $E$. For any $e \in E$, denote the $\varepsilon$-class containing $e$ by $\bar{e}$ or $E(e)$. Write $Y=\{\bar{e}: e \in E\}$. Since $E$ is left regular, then $E(e)$ is a left zero semigroup. Let $\gamma=\{(x, y) \in Q \times Q: V(x)=V(y)\}$. It is well known that $\gamma$ is the minimum inverse semigroup congruence on $Q$ and $\gamma \mid E=\varepsilon$. Suppose that $Q$ is a band of groups, then $Q / \gamma$ is a semilattice of groups and we can write $Q / \gamma=\bigcup_{\bar{e} \in Y} H_{\bar{e}}$ where $H_{\bar{e}}$ is the group $\mathscr{H}$-class in $Q / \gamma$ containing $\bar{e}$. Moreover, $Q$ is a spined product $P$ of $E$ and $Q / \gamma$, that is,

$$
P=\left\{\left(x^{-1} x, x \gamma\right) \in E \times Q / \gamma ; x \in Q\right\}
$$


We emphasize that $P$ is a semilattice of the direct products $E(e) \times H_{\bar{e}}, \bar{e} \in Y$ and the product of $P$ is reduced from the Cartesian product $E \times Q / \gamma$. Moreover, for any $(f, y \gamma)$ in $E(e) \times H_{\bar{e}} ;(f, y \gamma) \mathscr{H}(f, \bar{e})$ in $P$, the inverse of $(f, y \gamma)$ in $H_{(f, \bar{e})}$ is $\left(f, y^{-1} \gamma\right)$. We refer the reader to [1] and [12] for further details.

Let $S$ be a semigroup which has $P$ as a semigroup of left quotients. For any $\bar{e} \in Y$, define a subset $M_{\bar{e}}$ of $Q / \gamma$ by the rule; $m \in M_{\bar{e}}$ if and only if $m \in Q / \gamma$ and $(f, m) \in S$ for some $f \in E(e)$.

LeMma 3.1. For any $\bar{e}$ in $Y ; M_{\bar{e}}$ is a cancellative semigroup.

Proof. It is clear from Corollary 2.3 and the consideration of the subgroups of $P$ that $M_{\bar{e}}$ is not empty. It is also clear that $M_{\bar{e}}$ is a subsemigroup of $H_{\bar{e}}$ where $H_{\bar{e}}$ is the group $\mathscr{H}$-class in $Q / \gamma$. Hence $M_{\bar{e}}$ is cancellative.

Lemma 3.2. For any $\bar{e}$ in $Y, M_{\bar{e}}$ is right reversible.

Proof. Let $a \gamma, b \gamma$ be in $M_{\bar{e}}$ and $g, h$ in $E(e)$ so that $(g, a \gamma),(h, b \gamma)$ are in $S$. Choose $c \gamma \in M_{\bar{e}}$ and take $(k, c \gamma) \in S$ for some $k \in E(e)$. Since

$$
(k, c \gamma)(g, a \gamma)\left(h, b^{-1} \gamma\right) \in E(e) \times H_{\bar{e}},
$$

then by left ordering of $S$ in $P$, there exist $(f, q \gamma),(i, d \gamma)$ in $S$ and the inverse $\left(f, q^{-1} \gamma\right)$ of $(f, q \gamma)$ such that

$$
(f, q \gamma)(k, c \gamma)(g, a \gamma)(h, \bar{e})=(f, \bar{f})(i, d \gamma)(h, b \gamma)
$$

Hence $(g c) \gamma a \gamma=(f d) \gamma b \gamma$.

Now we show that $(g c) \gamma \in M_{\bar{e}}$ and $(f d) \gamma \in M_{\bar{e}}$. Notice from the first equality that $k=f i$ and thus $f k=k$. Since

$$
(f k,(q c) \gamma)=(f, q \gamma)(k, c \gamma) \in S, \quad \overline{f k}=\bar{k}=\bar{e},
$$

then $(q c) \gamma \in M_{\bar{e}}$.

Similarly, $(f i,(f d) \gamma)=(f, \bar{f})(i, d \gamma) \in S, \overline{f i}=\bar{k}=\bar{e}$ and $(f d) \gamma \in M_{\bar{e}}$.

Hence the result.

Now we put $M=\bigcup_{\bar{e} \in Y} M_{\bar{e}}, \quad M$ is a semilattice $Y$ of right reversible, cancellative semigroups $M_{\bar{e}}, e \in Y$. It is easy to see that $\bigcup_{\bar{e} \in Y}\left(E(e) \times M_{\bar{e}}\right)$ is a spined product containing $S$. Moreover, as a straightforward consequence of the fact that $S$ intersects every $\mathscr{H}$-class of $P$, we have

\section{LEMMA 3.3.}

(i) For any $e \in E$, there exists $x \gamma \in H_{\bar{e}}$ with $(e, x \gamma) \in S$.

(ii) For any $f \in E, y \gamma \in M_{\bar{e}}$, there exists $g \in E(f)$ with $(g, y \gamma) \in S$.

Now it follows that $S$ is a punched spined product and the following result is established.

Proposition 3.4. Let $P$ be a left regular band of groups and $S$ be a semigroup. If $P$ is a semigroup of quotients of $S$, then $S$ is a punched spined product of a left regular band and a semilattice of right reversible, cancellative semigroups. 
For a converse of Proposition 3.4, let $S$ be a punched spined product of a left regular band $E$ and a semilattice $Y$ of right reversible, cancellative semigroups $M \alpha, \alpha \in Y$. For $\alpha \in Y$, let $E_{\alpha}$ be the $J$-class of $E$ indexed by $\alpha$. For $\alpha \in Y$ and $e \in E_{\alpha}$, let

$$
M_{e, \alpha}=\left\{(e, x): x \in M_{\alpha}\right\} \quad \text { and } \quad S_{e, \alpha}=S \cap M_{e, \alpha}
$$

Since $S$ is a punched spined product, $S_{e, \alpha} \neq \varnothing$ and clearly $S_{e, \alpha}$ is a subsemigroup of $S$. Thus $S$ is a left regular band of cancellative semigroups $S_{e, \alpha}, \alpha \in Y, e \in E_{\alpha}$.

Let $\alpha \in Y, e \in E_{\alpha}$ and $(e, u),(e, v) \in S_{e, \alpha}$ As $M_{\alpha}$ is right reversible, there are elements $h, k \in M_{\alpha}$ with $h u=k v$. By the assumption, there are elements $f, g \in E_{\alpha}$ with $(f, h)(g, k) \in S$ and then;

$$
(e, u h)=(e, u)(f, h) \in S_{e, \alpha},(e, u k)=(e, u)(g, k) \in S_{e, \alpha}
$$

and further,

$$
(e, u h)(e, u)=(e, u h u)=(e, u k v)=(e, u k)(e, v),
$$

Thus $S_{e, \alpha}$ is right reversible.

Now by a direct application of Theorem 2.6 we get the converse of Proposition 3.4.

In conclusion we have the following result:

THEOREM 3.5. A semigroup $S$ has a left regular band of groups as a semigroup of left quotients if and only if $S$ is a punched spined product of a left regular band and $a$ semilattice of right reversible, cancellative semigroups.

The following Corollary is an immediate consequence of Theorem 3.5.

COROLlARY 3.6. If $S$ is a spined product of a left regular band and a semilattice of right reversible, cancellative semigroups, then $S$ has a left regular band of groups as a semigroup of left quotients.

For the rest of the section, let $S$ be a spined product of a left regular band $E$ and a semilattice $Y$ of cancellative semigroups $M_{\alpha} ; \alpha \in Y$. Put $E=\bigcup_{\alpha \in Y} E_{\alpha}, M=\bigcup_{\alpha \in Y} M_{\alpha}$ and $S=\bigcup_{\alpha \in Y}\left(E_{\alpha} \times M_{\alpha}\right)$.

Lemma 3.7. The relation $\mathscr{H}^{*}$ is the greatest semilattice congruence on $M$ all of whose classes are cancellative.

Proof. By Lemma 1.6, $\mathscr{H}^{*}$ is the greatest band congruence on $M$ all of whose classes are cancellative. The relation $\gamma$ defined on $M$ by the rule

$$
(a, b) \in \gamma \text { if and only if } a, b \in M_{\alpha} \text { for some } \alpha \in Y
$$

is a band congruence on $M$ all of whose classes are cancellative. Therefore, $\gamma \subseteq \mathscr{H}^{*}$. Now for any elements $a, b$ in $M$, we have $(a b, b a) \in \gamma$. Hence $a b \mathscr{H}^{*} b a$ and $M / \mathscr{H}^{*}$ is a semilattice.

Identify the semilattice $M / \mathscr{H}^{*}$ by $J$, that is, $M$ is a semilattice $J$ of $H_{j}^{*} ; j \in J$. For each $j \in J$, let $Z_{j}=\left\{\alpha \in Y ; M_{\alpha} \subseteq H_{j}^{*}\right\}$. Readily, $Z_{j}$ is a subsemilattice of $Y$, for any $j \in J$. Put $F_{j}=\bigcup_{\alpha \in Z_{j}} E_{\alpha}$ and $S_{j}=\bigcup_{\alpha \in Z_{j}}\left(E_{\alpha} \times M_{\alpha}\right)$.

We are now in a position to prove the final result of this section. 
Proposition 3.8. The following statements concerning the semigroup $S$ are equivalent:

(1) each $\mathscr{H}^{*}$-class of $M$ is right reversible.

(2) for any $a, b$ in $M$, there exist $x, y$ in $M$ with $x a=y b$ and $x \mathscr{H}^{*} y \mathscr{H}^{*} a b$.

(3) $S_{j}$ is right reversible for any $j$ in $j$.

(4) There is an oversemigroup $T$ of $S$ which is a left regular band $\mathscr{X}$ of right reversible, cancellative semigroups $T_{\alpha} ; a \in \chi$ where for any $j \in J ;{ }_{j} H^{*}$ is isomorphic to $T_{\alpha}$ for some $\alpha \in \chi$.

Proof. Recall that $\mathscr{H}^{*}$ is a semilattice congruence on $M$.

(1) $\Leftrightarrow(2)$ is (iii) $\Leftrightarrow$ (iv) of Theorem 5.1 of [7].

(1) $\Leftrightarrow(3)$ If (1 holds and $j \in J,(e, a),(f, b)$ in $S_{j}$, that $(e, a) \in E_{\alpha} \times M_{\alpha},(f, b) \in E_{\beta} \times$ $M_{\beta}$, say, where $M_{\alpha}$ and $M_{\beta}$ are subsets of $H_{j}^{*}$, then there exist $x, y \in H_{j}^{*}$ with $x a=y b$, where $x \in M_{\lambda}, y \in M_{\mu}$ for some $\lambda, \mu \in Z_{j}$. It follows that $\lambda_{\alpha}=\mu \beta$. Let $g \in E_{\lambda}, h \in E_{\mu}$ and $s \in M_{\lambda \alpha}=M_{\mu \beta}$. Then

$$
\text { gehf } \in E_{\lambda} E_{\alpha} E_{\mu} E_{\beta} \subseteq E_{\lambda \alpha}, \quad s x \in M_{\lambda \alpha M_{\lambda} \subseteq M_{\lambda \alpha}}, \quad s y \in M_{\mu \beta} M_{\beta} \subseteq M_{\mu \beta}
$$

and, $s x a=s y b$. The elements

$$
\left(\text { gehf, sx ), (gehf, sy) are in }\left(E_{\alpha} \times M_{\alpha}\right)\right.
$$

so that, they are in $S_{j}$. Moreover,

$$
(g e h f, s x a)=(\text { gehf, syb), that is, }(\text { gehfe, sxa })=(\text { gehff, syb })
$$

and $(g e h f, s x)(e, a)=(g e h f, s y)(f, b)$. Hence (3) holds.

If (3) holds and $a, b$ in $H_{j}^{*}$, then for some $\alpha, \beta \in Z_{j}, a \in M_{\alpha}, b \in M_{\beta}$. Let $e \in E_{\alpha}$, $f \in E_{\beta}$ so that $(e, a),(f, b)$ are in $S_{j}$. Then there exist $(g, x),(h, y)$ in $S_{j}$ with $(g, x)(e, a)=(h, y)(f, b)$. In particular; $x, y \in H_{j}^{*}, x a=y b$ and (1) holds.

$(1) \Leftrightarrow(4)$ if (1) holds, then by Lemma 3.7, $H_{j}^{*}$ and hence $\{e\} \times H_{j}^{*}$ is a right reversible, cancellative semigroup for any $e \in E_{\alpha}, \alpha \in Z_{j}$. For any $j \in J, \alpha \in Z_{j}$, put

$$
N_{\alpha}=\bigcup_{e \in E_{\alpha}}\left(\{e\} \times H_{j}^{*}\right) \text { so that } F_{j} \times H_{j}^{*}=\bigcup_{\alpha \in Z_{j}} N_{\alpha}
$$

and

$$
\begin{aligned}
T & =\bigcup_{j \in J}\left(F_{j} \times H_{j}^{*}\right)=\bigcup_{j \in J}\left(\bigcup_{\alpha \in Z_{j}} N_{\alpha}\right) \\
& =\bigcup_{j \in J}\left(\bigcup_{\alpha \in Z_{j}}\left(\bigcup_{e \in E_{\alpha}}\left(\{e\} \times H_{j}^{*}\right)\right)\right)
\end{aligned}
$$

is a left regular band of right reversible, cancellative semigroups. Clearly, for any $j \in J$, $\alpha \in Z_{j}, e \in E_{\alpha} ;\{e\} \times H_{j}^{*} \approx H_{j}^{*}$ and $S$ is a subsemigroup of $T$. Hence (4) holds.

If (4) holds, then trivially (1) holds.

4. $\mathscr{R}^{*}$-unipotent semigroups. Recall from (4) that a semigroup $S$ is abundant if each $\mathscr{R}^{*}$-class and each $\mathscr{L}^{*}$-class of $S$ contains an idempotent. If $a$ is an element of $S$, the $a^{+}$ and $a^{*}$ denote typical idempotents in $\mathscr{R}_{a}^{*}$ and $L_{a}^{*}$ respectively. A semigroup $S$ is superabundant if each $\mathscr{H}^{*}$-class contains an idempotent. In this section we consider the class of abundant semigroups $S$ in which the set of idempotents forms a left regular band. In this case every $\mathscr{R}^{*}$-class of $S$ contains a unique idempotent, thus $S$ is called 
$\mathscr{R}^{*}$-unipotent. The objective is to characterize a class of $\mathscr{R}^{*}$-unipotent semigroups which have a semigroup $Q$ of left quotients, where $Q$ is a left regular band of groups. This is a special case of the subject discussed in the previous sections. The dual of $\mathscr{R}^{*}$-unipotent semigroups was studied in [3] from which we conclude the following result.

LemmA 4.1. Let $S$ be an $\mathscr{R}^{*}$-unipotent semigroup. Then

(1) $S$ is superabundant if and only if $\mathscr{R}^{*}=\mathscr{H}^{*}$ on $S$.

(2) $S$ is a band of cancellative monoids if and only if $S$ is superabundant and $\mathscr{L}^{*}$ is a congruence on $S$.

Throughout this section, let $S$ be an $\mathscr{R}^{*}$-unipotent semigroup.

Proposition 4.2. If $S$ is a left regular band $Y$ of right reversible, cancellative semigroups $S_{\alpha}, \alpha \in Y$, then the following statements are equivalent

(1) $S$ is superabundant

(2) for any $\alpha \in Y, a \in S_{\alpha}$, there exists an idempotent $e_{\gamma}$ in $S_{\gamma}$ for some $\gamma \in Y$ with $e \mathscr{L}^{*} a$ and $\gamma \alpha=\alpha$.

Proof. (1) $\Rightarrow$ (2) Let $\alpha \in Y, a \in S_{\alpha}$ and $a \mathscr{R}^{*} e_{\gamma}$ where $e_{\gamma}$ is an idempotent in $S_{\gamma}$. Since $\mathscr{R}^{*}=\mathscr{H}^{*}$ on $S$ (Lemma 4.1) then $a \mathscr{L}^{*} e_{\gamma}$ and $e_{\gamma} a=a$, that is $S_{\gamma} S_{\alpha} \subseteq S_{\alpha}$ so that $\gamma \alpha=\alpha$.

(2) $\Rightarrow$ (1) Let $a \in S_{\alpha}$ where $a \mathscr{R}^{*} e_{\delta}, e_{\delta}$ is an idempotent in $S_{\delta}$. Then $e_{\delta} a=a$, that is, $\delta \alpha=\alpha$. It follows that $\alpha \delta \alpha=\alpha$ and $\alpha \delta=\alpha$. In particular $a e_{\delta} \in S_{\alpha}$. By right reversibility of $S_{\alpha}, x a=y a e_{\delta}$ for some $x, y \in S_{\alpha}$, so that $x a e_{\delta}=y a e_{\delta}$. The cancellation in $S_{\alpha}$ implies $x=y$ and $x a=x a e_{\delta}$. Thus $a=a e_{\delta}$. Now let $e_{\gamma}$ be an idempotent in $S_{\gamma}$ with $e \mathscr{L}^{*} a$ and $S_{\gamma} S_{\alpha} \subseteq S_{\alpha}$. Since $a e_{\gamma}=a=a e_{\delta}$ and $e \mathscr{L}^{*} a$, then $e_{\gamma}=e_{\gamma} e_{\delta}$. Recall that $e_{\gamma} a \in S_{\gamma} S_{\alpha} \subseteq S_{\alpha}$, $a \in S_{\alpha}, S_{\alpha}$ is right reversible so that $u e_{\gamma} a=v a$ for some $u, v \in S_{\alpha}$. Since $a \mathscr{R}^{*} e_{\delta}$, we get $u e_{\gamma} e_{\delta}=v e_{\delta}$. But $e_{\gamma} e_{\delta}=e_{\gamma}$ and $v e_{\delta}=v e_{\alpha} e_{\delta}=v e_{\alpha}=v$. Thus $u e_{\gamma}=v$. Therefore, $u e_{\gamma} e_{\gamma} a=$ $v a$ and $e_{\gamma} a=a$. Since $e_{\gamma} a=a=e_{\delta} a$ and $e_{\delta} \mathscr{R}^{*} a$, then $e_{\gamma} e_{\delta}=e_{\delta}$. Hence $e_{\gamma}=e_{\delta}$ and $a \mathscr{L}^{*} e_{\delta}$, that is, $a H^{*} e_{\delta}$ and (1) holds.

LEMMA 4.3. If $S$ is superabundant in which for any elements $a, b$ in $S$ there exist $x, y$ in $S$ with $x a=y b$ and $x \mathscr{H}^{*} y \mathscr{H}^{*} a b$, then each $\mathscr{H}^{*}$-class in $S$ is right reversible.

Proof. This is immediate from the fact that each $\mathscr{C}^{*}$-class of $S$ is a cancellative monoid.

Proposition 4.4. If $S$ is a band of cancellative monoids, then the following statements are equivalent

(1) each $\mathscr{H}^{*}$-class in $S$ is right reversible.

(2) for any $a, b$ in $S$, there exist elements $x, y$ in $S$ with $x a=y b$ and $x \mathscr{H}^{*} \mathscr{H}^{*} a b$.

Proof.

$(1) \Rightarrow(2)$ By Lemma (4.1), $S$ is superabundant on which $\mathscr{H}^{*}$ is a congruence. Let $a \in H_{e}^{*}, b \in H_{f}^{*}$ for some idempotents, $e, f$ in $S$. Then

$$
a b \in H_{e f}^{*}, \quad a b a \in H_{e f e}^{*}=H_{e f}^{*}
$$

But $H_{e f}^{*}$ is right reversible, so there exist, $u, v$ in $H_{e f}^{*}$ such that $u a b=v a b a$. Notice that $y=u a \in H_{e f e}^{*}=H_{e f}^{*}$ and $x=v a b \in H_{e f f}^{*}=H_{e f}^{*}$. Therefore

$$
x a=y b \text { and } x \mathscr{H}^{*} y H^{*} a b .
$$

$(2) \Rightarrow(1)$ This is Lemma 4.3 . 
In fact any of the statements of Proposition 4.4 is a consequence of $S$ to have a semigroup $Q$ of left quotients where $Q$ is a left regular band of groups. The following lemma demonstrates this result.

LEMMA 4.5. Let $S$ be superabundant which is a left regular band of right reversible, cancellative semigroups. Then for any elements $a, b$ of $S$, there exist $x, y$ in $S$ with $x a=y b$ and $x \mathscr{H}^{*} y \mathscr{H}^{*} a b$.

Proof. Put $S=\bigcup_{\alpha \in Y} S_{\alpha}$, where $Y$ is a left regular band and $S_{\alpha}$ is a right reversible, cancellative semigroup for any $\alpha \in Y$. Let $a, b \in S ; a \in S_{\alpha}, b \in S_{\beta}$, say. Then $a b \in S_{\alpha \beta}$, $a b a \in S_{\alpha \beta \alpha}=S_{\alpha \beta}$ and there exist $u, v$ in $S_{\alpha \beta}$ with $u a b a=v a b$ where

$$
x=u a b \in S_{\alpha \beta \alpha \beta}=S_{\alpha \beta} \text { and } y=v a \in S_{\alpha \beta \alpha}=S_{\alpha \beta}
$$

But every two elements in $S_{\alpha \beta}$ are $\mathscr{R}^{*}$-related (Lemma 2.4). Then the result follows from the fact that $\mathscr{R}^{*}=\mathscr{H}^{*}$ on $S$.

Now we consider the construction of $S_{\alpha}$ in $S$ as given in the following proposition.

Proposition [3] 4.6. Let $S$ be superabundant with band of idempotents $E$ and $E=\bigcup_{\alpha \in Y} E_{\alpha}$ be the maximal semilattice decomposition of $E$. For each $\alpha \in Y$, define

$$
S_{\alpha}=\left\{x \in S: x^{+}, x^{*} \in E_{\alpha}\right\}
$$

\section{Then}

(1) $S_{\alpha}$ is the maximal abundant semigroup of $S$ which contains $E_{\alpha}$ as its set of idempotents such that $\mathscr{R}^{*}\left(S_{\alpha}\right) \subseteq \mathscr{R}^{*}(S)$ and $\mathscr{L}^{*}\left(S_{\alpha}\right) \subseteq \mathscr{L}^{*}(S)$

(2) $S_{\alpha} \cap S_{\beta}=\varnothing$ if $\alpha \neq \beta$

(3) $S$ is a semilattice of $S_{\alpha} ; \alpha \in Y$

(4) $S_{\alpha} \approx E_{\alpha} \times H_{e}^{*}$ where $H_{e}^{*}$ is the $\mathscr{H}^{*}$-class in $S$ containing $e$ and $e \in E_{\alpha}$

Now let $S$ be superabundant with set of idempotent $E$. Retain the notations of Proposition 4.6. Assign to each $\alpha$ in $Y$, a cancellative monoid $M_{\alpha}=H_{e}^{*}$ for some fixed $e$ in $E_{\alpha}$. By Lemma $1.4, M_{\alpha} \approx H_{f}^{*}$ for any $f \in E_{\alpha}$. By Proposition 4.6, $S_{\alpha} \approx E_{\alpha} \times M_{\alpha}$. Denote the identity of $M_{\alpha}$ by $e_{\alpha}$ and put $M=\bigcup_{\alpha \in Y} M_{\alpha}$. Define a product . on $M$ by

$$
x . y=e_{\alpha \beta} x y \text { for any } x \in M_{\alpha}, y \in M_{\beta}
$$

It is routine to check that $x, y \in M_{\alpha \beta}$ for any $x \in M_{\beta}$ and the product is a well-defined associative binary operation makes $M$ a semilattice $Y$ of the cancellative monoids $M_{\alpha}, \alpha \in Y$. Moreover, we have the following result

LEMMA 4.7. $S$ is in a one-to-one correspondence with $P=\bigcup_{\alpha \in Y}\left(E_{\alpha} \times M_{\alpha}\right)$.

Proof. Define $\Phi: P \rightarrow S$ by $(e, a) \Phi=e a$. It is obvious that $\Phi$ is a well-defined map. Let $(e, x) \in E_{\alpha} \times M_{\alpha},(f, y) \in E_{\beta} \times M_{\beta}$ such that $e x=f y$. It is easy to verify that $e \mathscr{R}^{*} e x$ and $f \mathscr{R}^{*} f y$. Therefore $e=f$ and $E_{\alpha}=E_{\beta}$, that is, $\alpha=\beta$. Thus

$$
e x=f y \Rightarrow e_{\alpha} e x=e_{\alpha} f y \Rightarrow e_{\alpha} x=e_{\alpha} y \Rightarrow x=y
$$

and $\Phi$ is one-to-one. For surjectivity, let $x \in S$ where $x \mathscr{R}^{*} x^{+} ; x^{+} \in E_{\alpha}$, say. Then $\left(x^{+}, e_{\alpha} x\right) \in E_{\alpha} \times M_{\alpha}$ and $\left(x^{+}, e_{\alpha} x\right) \Phi=x^{+} e_{\alpha} x=x^{+} x=x$.

Hence $\Phi$ is a bijection. 
Recall that a band $E$ is left normal if $e f g=e g f$ for any idempotents $e, f, g$, in $E$. Clearly left normal bands are left regular. To improve the result of Lemma 4.7 we impose the condition of left normality on $E$.

Proposition 4.8. If $E$ is left normal, then $P=\bigcup_{\alpha \in Y}\left(E_{\alpha} \times M_{\alpha}\right)$ is isomorphic to $S$.

Proof. From the proof of Lemma 4.7 we have the bijection $\Phi: P \rightarrow S$, defined by $(e, a) \Phi=e a$ for any $(e, a) \in P$. To show that $\Phi$ is a homomorphism, let $(e, x) \in E_{\alpha} \times M_{\alpha}$ and $(f, y) \in E_{\beta} \times M_{\beta}$. Then

$$
\begin{array}{rlr}
((e, x) .(f, y)) \Phi & =\left(e f, e_{\alpha \beta} x y\right) \Phi & \\
& =e f e_{\alpha \beta} x y \\
& =e f x y \quad\left(e f, e_{\alpha \beta} \in E_{\alpha \beta}\right)
\end{array}
$$

and

$$
(e, x) \Phi(f, y) \Phi \Rightarrow e x f y
$$

Notice that $e x \mathscr{R}^{*} e$ and

$$
\begin{aligned}
\text { ex: } \mathscr{R}^{*} e & \Rightarrow \text { efex } \mathscr{R}^{*} e f e \\
& \Rightarrow \text { efex } \mathscr{R}^{*} e f \\
& \Rightarrow \text { efex } \mathscr{L}^{*} \text { ef } \quad\left(\mathscr{R}^{*}=\mathscr{H}^{*} \text { on } S\right) \\
& \Rightarrow \text { efexef }=e f x \\
& \Rightarrow \text { efexfy }=e f x y \\
& \Rightarrow \text { efxfy }=e f x y
\end{aligned}
$$

Now let $i \in E$ such that $x \mathscr{H}^{*} i$. Then in particular we have $x f i=x f$, that is $x f i=x f f$ which implies $i=$ if. Therefore

$$
\begin{aligned}
\text { efxfy } & =\text { efixfy } & & \\
& =\text { eif } x y \quad & & (E \text { is left normal }) \\
& =\text { eixfy } & & (\text { if }=i) \\
& =\text { exfy } & &
\end{aligned}
$$

and we obtain exfy $=e f x y$. Hence $\Phi$ is an isomorphism.

As an immediate consequence of Proposition 4.8 we have the following Corollary.

COROLLARY 4.9. If $E$ is left normal, then $S$ is a spined product of a left regular band and a semilattice $Y$ of cancellative monoids $M_{\alpha} ; \alpha \in Y$ where $M_{\alpha}$ 's are $\mathscr{H}^{*}$-classes of $S$.

Now directly from Theorem 2.6, Lemma 4.5, Lemma 4.3, Corollary 4.9 and Corollary 3.7 we have the following result.

THEOREM 4.10. Let $S$ be superabundant in which the set of idempotents is a left normal band. Then the following statements are equivalent. 
(1) $S$ is a left order in a left normal band of groups.

(2) $S$ is a left order in a left regular band of groups.

(3) $S$ is a left regular band of right reversible, cancellative semigroups.

(4) For any $a, b$ in $S$, there exist $x, y$ in $S$ with $x a=y b$ and $x \mathscr{H}^{*} y \mathscr{H}^{*} a b$.

(5) Each $\mathscr{H}^{*}$-class in $S$ is right reversible.

(6) $S$ is a spined product of a left regular band and a semilattice of right reversible, cancellative semigroups.

Acknowledgements. The Author would like to thank Professor Abdus Salam, the International Atomic Energy Agency and UNESCO for hospitality at the International Centre for Theoretical Physics, Trieste. He would also like to thank the referee of this paper for his valuable suggestions.

\section{REFERENCES}

1. G. Bailes, Right inverse semigroups, J. Algebra 26 (1973), 492-507.

2. A. H. Clifford and G. B. Preston, The algebraic theory of semigroups, Mathematical Surveys 7 (Vol. 1), Amer. Math. Soc. (Providence, Rhode Island, 1961).

3. A. El-Qallali, $\mathscr{L}^{*}$-unipotent semigroups, J. Pure Appl. Algebra, 62 (1989), 19-33.

4. J. B. Fountain, Abundant semigroups, Proc. London Math. Soc. (3) 44 (1982), 103-129.

5. J. B. Fountain and M. Petrich, Completely 0-simple semigroups of quotients, J. Algebra 101 (1986), 365-402.

6. V. Gould, Bisimple inverse-semigroups of left quotients, Proc. London Math. Soc. (3) 52 (1986), 95-118.

7. V. Gould, Clifford semigroups of left quotients, Glasgow Math. J. 28 (1986), 181-191.

8. V. Gould, Orders in semigroups, Contributions to general algebra V (Verlag HölderPichler-Tempsky, 1987) 163-169.

9. J. M. Howie, An introduction to semigroup theory (Academic Press, 1976).

10. D. B. McAllister, One-to-one partial right translations of a right cancellative semigroups, J. Algebra 43 (1976), 231-251.

11. F. Pastijn, A representation of a semigroup by a semigroup of matrices over a group with zero, Semigroup Forum 10 (1975), 238-249.

12. M. Petrich, Regular semigroups which are subdirect products of a band and a semilattice of groups, Glasgow Math. J. 14 (1973), 27-49.

13. P. S. Venkatesan, On right unipotent semigroups, Pacific J. Math. 63(2) (1976), 555-561.

14. H. J. Weinert, On S-sets and semigroups of quotients, Semigroup Forum 19 (1980), 1-78.

Department of Mathematics,

AL-FATEH UNIVERSITY,

TRIPOLI,

LYBIA. 